\title{
A!
}

This is an electronic reprint of the original article.

This reprint may differ from the original in pagination and typographic detail.

Mironov, S. V.; Mel'Nikov, A. S.; Tokman, I. D.; Vadimov, V.; Lounis, B.; Buzdin, A. I.

\section{Inverse Faraday Effect for Superconducting Condensates}

Published in:

Physical Review Letters

DOI:

10.1103/PhysRevLett.126.137002

Published: 02/04/2021

Document Version

Publisher's PDF, also known as Version of record

Please cite the original version:

Mironov, S. V., Mel'Nikov, A. S., Tokman, I. D., Vadimov, V., Lounis, B., \& Buzdin, A. I. (2021). Inverse Faraday Effect for Superconducting Condensates. Physical Review Letters, 126(13), [137002].

https://doi.org/10.1103/PhysRevLett.126.137002

This material is protected by copyright and other intellectual property rights, and duplication or sale of all or part of any of the repository collections is not permitted, except that material may be duplicated by you for your research use or educational purposes in electronic or print form. You must obtain permission for any other use. Electronic or print copies may not be offered, whether for sale or otherwise to anyone who is not an authorised user. 


\title{
Inverse Faraday Effect for Superconducting Condensates
}

\author{
S. V. Mironov ${ }^{1},{ }^{1}$ A. S. Mel'nikov, ${ }^{1,2}$ I. D. Tokman, ${ }^{1}$ V. Vadimov $\odot,{ }^{1,3}$ B. Lounis, ${ }^{4,5}$ and A. I. Buzdin $\circledast^{2,6}$ \\ ${ }^{1}$ Institute for Physics of Microstructures, Russian Academy of Sciences, 603950 Nizhny Novgorod, GSP-105, Russia \\ ${ }^{2}$ University Bordeaux, LOMA UMR-CNRS 5798, F-33405 Talence Cedex, France \\ ${ }^{3}$ Department of Applied Physics, QCD Labs and MSP Group, QTF Centre of Excellence, Aalto University, \\ P.O. Box 15100, FI-00076 Aalto, Espoo, Finland \\ ${ }^{4}$ Institut d'Optique, LP2N UMR-CNRS 5298, F-33400 Talence, France \\ ${ }^{5}$ University Bordeaux, LP2N, F-33400 Talence, France \\ ${ }^{6}$ World-Class Research Center "Digital Biodesign and Personalized Healthcare”, Sechenov First Moscow State Medical University, \\ Moscow 119991, Russia
}

(Received 8 October 2020; accepted 1 March 2021; published 2 April 2021)

\begin{abstract}
The Cooper pairs in superconducting condensates are shown to acquire a temperature-dependent dc magnetic moment under the effect of the circularly polarized electromagnetic radiation. The mechanisms of this inverse Faraday effect are investigated within the simplest version of the phenomenological dynamic theory for superfluids, namely, the time-dependent Ginzburg-Landau (GL) model. The light-induced magnetic moment is shown to be strongly affected by the nondissipative oscillatory contribution to the superconducting order parameter dynamics, which appears due to the nonzero imaginary part of the GL relaxation time. The relevance of the latter quantity to the Hall effect in the superconducting state allows us to establish the connection between the direct and inverse Faraday phenomena.
\end{abstract}

DOI: 10.1103/PhysRevLett.126.137002

The exploration of mechanisms allowing the generation and control of magnetic moment in solids solely by light has always been an attractive challenge for condensed matter physics. The first systematic studies of the interplay between magnetism and optics are dated back to the works of Faraday, who discovered the rotation of the plane of the light polarization by a magnetic field. The inverse effect, namely, the generation of dc magnetic moment by the circularly polarized light (so-called inverse Faraday effect) was predicted lately by Pitaevskii [1] and then observed in nonabsorbing $\mathrm{Eu}^{+3}: \mathrm{CaF}$ garnet [2]. Currently, the interaction of the very short laser pulses with nonabsorbing media is a rapidly expanding research topic of modern magnetism [3,4], and it has been clearly demonstrated that for the insulating magnetic materials the subpicosecond polarized laser pulses provide a tool for the magnetic moment manipulation at the femtosecond timescale [5].

In contrast, the optical generation of the magnetic moment in conductive materials still remains challenging. There are several experimental hints supporting the observation of the inverse Faraday effect (IFE) in GdFeCo magnetic metallic amorphous alloy [6], however, up to now the evidence of the IFE in nonmagnetic metals has been reported only in a few publications (see, e.g., [7]). Theoretical works are also rather scarce in this domain: the estimates of the IFE in metal plasma have been suggested in [8-10], a very simplified "harmonic atom" model has been studied in [11], the case of semiconductors has been approached in [12], and persistent currents appearing in ballistic nanorings due to the IFE were treated in $[13,14]$. Despite the strong differences in the electronic band structure of these materials, the physics beyond the IFE in conductive finite-size samples is rather generic. The electric field $\mathbf{E}$ of the circularly polarized wave propagating perpendicular to the sample surface induces the excess charge density $\rho$ at the sample edges. The spatial rotation of the vector $\mathbf{E}$ produces the corresponding in-phase motion of the charge $\rho$ with the velocity $\mathbf{v}$ along the sample boundary, which results in the nonzero time-averaged edge current $\mathbf{j}=\langle\rho \mathbf{v}\rangle$ and, thus, the emergence of dc magnetic moment.

During the past decade, it became possible to study the IFE in artificial superfluid systems. In particular, it was experimentally demonstrated that illumination of the toroidal atomic Bose-Einstein condensate by the twisted light carrying nonzero angular momentum produces dc persistent supercurrents $[15,16]$. The superfluids seem to provide a promising playground to study the IFE since the optically induced supercurrents should survive even after switching off the light. One can expect that similar light-stimulated persistent currents should emerge also in conventional solid-state superconductors, which may open the way for the ultrafast optical control of magnetic states in the devices of superconducting spintronics [17]. However, at the moment, both the theory and experiment dealing with the IFE in superconductors is lacking.

It is the goal of the present Letter to suggest a theoretical description of the IFE in superconductors, which may help 


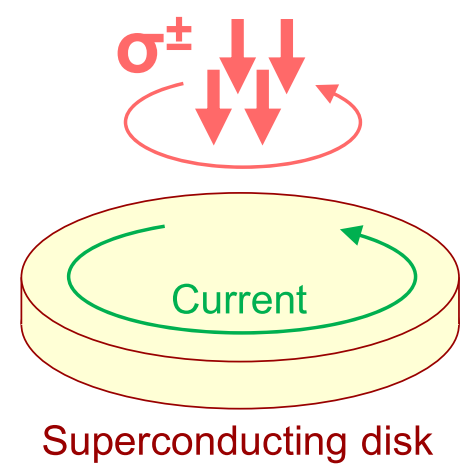

FIG. 1. Sketch of the thin superconducting disk radiated by the circularly polarized electromagnetic wave with two different polarizations $\left(\sigma^{ \pm}\right)$. The radiation-induced edge currents generate the magnetic moment of the disk (inverse Faraday effect).

to elaborate an appropriate experimental setup needed to observe the light-stimulated magnetic states in superconducting systems (see the exemplary setup in Fig. 1). To elucidate the key ideas of our work, we start from the qualitative consideration of the specific features of the IFE in superconductors. The first obvious difference between the IFE in normal metals and superconductors is based on the difference in dc magnetic response. Indeed, the magnetic response of the nonferromagnetic normal metal to the dc magnetic moment $M_{0}$ generated by the electromagnetic radiation is determined by the Pauli and Landau terms in the susceptibility and both these terms are known to be extremely small [18]. In the opposite, the magnetic moment in the superconductor should be screened by the Meissner supercurrents. The screening can be partial or full depending on the sample geometry and the ratio of the its size to the London penetration depth. As a result, the total dc magnetic moment in the superconducting state can be partially or fully suppressed by the screening currents. This compensation will be broken when we increase the induced magnetic moment due to the vortex entry into the sample. To sum up, the overall picture is very similar to the one in the ferromagnetic superconductors [19] if we take account only of the Meissner screening phenomenon. These screening effects do not relate to the physical mechanisms of the $M_{0}$ formation, though they may be important for the particular experimental setup and system configuration.

Focusing now on the physics of formation of the primary magnetic moment $M_{0}$, one can take a general relation between the current density and vector potential $\mathbf{j}(t)=\hat{Q}(N, \Delta) \mathbf{A}(t)$, where the integral operator $\hat{Q}$ depends on the electron concentration $N$ and superconducting gap $\Delta$ and also accounts for the relevant time dispersion. According to Ref. [8] the IFE can be obtained if we consider the second-order nonlinear corrections in the field of the electromagnetic wave in the above material relation. Thus, we need the corrections to the operator $\hat{Q}$ linear in the electric field $\mathbf{E}$. These corrections may originate from the deviations in the local concentration $\delta N \propto \operatorname{div} \mathbf{E}$ from its equilibrium value $N_{0}$. The simplest expansion for the current density that assumes the local in time dependence on the deviation $\delta N(t)$ reads: $\mathbf{j}(t)=\hat{Q}_{0} \mathbf{A}(t)+\left[\delta N(t) / N_{0}\right] \hat{Q}_{1} \mathbf{A}(t)$. In the frequency representation (for the $e^{-i \omega t}$ processes) the operator $\hat{Q}_{1}$ can be written as follows:

$$
Q_{1}(\omega)=N_{0}\left(\frac{\partial Q_{0}(\omega)}{\partial N}+\frac{\partial Q_{0}(\omega)}{\partial \Delta} \frac{\partial \Delta}{\partial \mu} \frac{\partial \mu}{\partial N}\right)
$$

where $Q_{0}(\omega)$ denotes the frequency-dependent linear response of the system and $\mu$ is the chemical potential. We estimate $\left(\partial Q_{0} / \partial N\right) \sim Q_{0} / N_{0}, \quad(\partial \Delta / \partial \mu) \sim \Delta / E_{F}$, $(\partial \mu / \partial N) \sim \lambda_{T F}^{2} e^{2}$, where $E_{F}$ is the Fermi energy, and $\lambda_{T F}$ is the Thomas-Fermi screening length. Finally, we get

$$
Q_{1}(\omega) \sim Q_{0}+\Delta \frac{\partial Q_{0}}{\partial \Delta}\left(k_{F}^{2} \lambda_{T F}^{2}\right) \frac{k_{F} e^{2}}{E_{F}},
$$

where $k_{F}$ is the Fermi momentum. The first term in this expression is similar to the one obtained by Hertel in [8] for the normal metals, though, of course, it takes account of the full response of both nonsuperconducting and superconducting carriers. The second term is specific for superconductors and reflects the concentration dependence of the superconducting gap function. Clearly this term is nonzero only provided we take account of the gap dynamics directly induced by the incident electromagnetic wave. Despite the presence of the small parameter $\Delta / E_{F}$ in one of the two contributions to Eq. (2), the final estimate shows that both contributions to the $Q_{1}$ value can be comparable, though their relation depends certainly on temperature. Specifically, at temperature slightly below the superconducting transition temperature $T_{c}$, the dependence $Q_{0}(\Delta)$ is power law and $\Delta\left(\partial Q_{0} / \partial \Delta\right) \sim Q_{s}$, where $Q_{s}$ is the part of the response function determined by the contribution of superconducting carriers. At low temperatures $T \rightarrow 0$, the derivative $\partial Q_{0} / \partial \Delta$ vanishes, and thus the maximum value of the second term in Eq. (2) is reached at intermediate temperatures. Taking now the expression for $\delta N(\omega)=-\operatorname{div} \mathbf{j} / i \omega e=-\operatorname{div}\left[Q_{0} c \mathbf{E}(\omega) / \omega^{2} e\right]$ from the continuity equation, one can get the nonlinear contribution to the current density at zero frequency $\mathbf{j}_{1}=\operatorname{rot} \mathbf{M}_{0}$, where the magnetic moment is given by the vector product

$$
\mathbf{M}_{0}=-\frac{i\left[Q_{0} \mathbf{E} \times Q_{1}^{*} \mathbf{E}^{*}\right] c^{2}}{N_{0} e^{2} \omega^{3}} .
$$

It is important to note that here the electric field $\mathbf{E}$ is taken inside the sample and its relation to the external field of the incident electromagnetic wave should be found from the solution of the linear scattering problem.

The above simple reasoning shows that the qualitatively new physics of the IFE in superconductors can arise only from the gap modulation effect associated with the second 
term in Eq. (2). In order to separate this effect from the other possible contributions, we choose a specific sample geometry, namely, a thin disk of the radius $R$, much smaller than both the superconducting screening length and the light wavelength (see Fig. 1). For simplicity we also assume this radius to be less than the length $l_{E}$ of the relaxation of the electron-hole imbalance potential, thus neglecting the possible conversion between the superconducting and normal currents. For the quantitative consideration of the gap modulation effect, we take the simplest phenomenological model known to describe the dynamics of the superconducting order parameter $\psi$ at rather low frequencies and based on the so-called time-dependent Ginzburg-Landau (GL) equation

$$
(\pi \alpha / 8+i \gamma) \hbar \partial_{t} \psi+\alpha T_{c} \epsilon \psi+\xi_{0}^{2} \hat{\mathbf{D}}^{2} \psi+b|\psi|^{2} \psi=0,
$$

where $\hat{\mathbf{D}}=-i \nabla+\left(2 \pi / \Phi_{0}\right) \mathbf{A}, \Phi_{0}=\pi \hbar c / e(e>0)$ is the magnetic flux quantum, $\xi_{0}$ is the superconducting zerotemperature coherence length, and $\epsilon=T / T_{c}-1$. Considering the electric field $\mathbf{E}=E_{0} \operatorname{Re}\left[\left(\mathbf{e}_{x}+i \mathbf{e}_{y}\right) e^{-i \omega t}\right]$ inside the disk we may choose the corresponding vector potential in the form $\mathbf{A}=\left(c E_{0} / \omega\right) \operatorname{Re}\left[\left(\mathbf{e}_{y}-i \mathbf{e}_{x}\right) e^{-i \omega t}\right]$ (here we choose the origin of the coordinate system with the in-plane axes $x$ and $y$ in the disk center). Here we assume the disk thickness $L$ to be much smaller than the skin depth and neglect the variation of the vector potential along the $z$ axis. Though the above model has a rather restricted range of validity and assumes a gapless superconducting state, such consideration is known to provide instructive insights for a great variety of dynamic phenomena (see $[20,21]$ for review). The key ingredient of Eq. (4) responsible for the IFE is the imaginary part of the dimensionless relaxation constant $\gamma \sim \alpha\left(T_{c} / E_{F}\right)$ arising due to electron-hole asymmetry [21]. In addition, one needs to impose the boundary conditions at the disk edge, which guarantee $\partial|\psi| / \partial r=0$ in the cylindrical coordinates $(r, \theta)$ and the absence of the radial superconducting current at $r=R$. The solution of Eq. (4) determines the superconducting current flowing in the disk,

$$
\mathbf{j}_{s}=\frac{2 \pi \alpha T_{c} \xi_{0}^{2} c}{\Phi_{0}}\left(i \psi^{*} \nabla \psi-i \psi \nabla \psi^{*}-\frac{4 \pi}{\Phi_{0}}|\psi|^{2} \mathbf{A}\right) .
$$

Note that since $R \ll l_{E}$, Eq. (4) does not contain the electrochemical potential.

Further, it is convenient to introduce the absolute value $\Delta$ and the phase $\chi$ of the order parameter: $\psi=\Delta \exp (i \chi)$. In the absence of radiation, the superconducting gap can be chosen real and equal to $\Delta_{0}^{2}=-\left(\alpha T_{c} / b\right) \epsilon$. The vector potential of the incident wave results in the corrections $\Delta_{1} \propto A$ and $\chi \propto A$ to the gap value and the superconducting phase, respectively. Introducing the temperature-dependent correlation length $\xi=\xi_{0} / \sqrt{|\epsilon|}$ $(\epsilon<0)$, the GL time $\tau=\pi \hbar / 8 T_{c}|\epsilon|$, and the small para- meter $\nu=8 \gamma / \pi \alpha \propto\left(T_{c} / E_{F}\right) \ll 1$, we obtain the first-order equations for $\Delta_{1}$ and $\chi$ together with the boundary conditions

$$
\begin{gathered}
\tau \frac{\partial \Delta_{1}}{\partial t}-\nu \tau \Delta_{0} \frac{\partial \chi}{\partial t}+2 \Delta_{1}-\xi^{2} \nabla^{2} \Delta_{1}=0, \\
\tau \Delta_{0} \frac{\partial \chi}{\partial t}+\nu \tau \frac{\partial \Delta_{1}}{\partial t}-\xi^{2} \Delta_{0} \nabla^{2} \chi=0 . \\
\left.\frac{\partial \Delta_{1}}{\partial r}\right|_{r=R}=0,\left.\left(\frac{\partial \chi}{\partial r}+\frac{2 \pi}{\Phi_{0}} A_{r}\right)\right|_{r=R}=0 .
\end{gathered}
$$

Note that the vector potential A controls only the boundary condition. Since the system (6)-(8) is linear, it is convenient to introduce the complex amplitudes $\tilde{u}: u=\operatorname{Re}\left(\tilde{u} e^{i \theta-i \omega t}\right)$, where $u=\left\{\Delta_{1}, \chi, \mathbf{A}\right\}$. Then the solution for the $\tilde{\Delta_{1}}$ and $\tilde{\chi}$, which accounts for only the corrections up to $O(\nu)$, reads [22]

$$
\begin{gathered}
\tilde{\chi}(r)=\frac{2 \pi i R}{\Phi_{0}} \frac{c E_{0}}{\omega} f\left(q_{2}, r\right), \\
\tilde{\Delta}_{1}(r)=\Delta_{0} \frac{\pi R c E_{0} \nu \tau}{\Phi_{0}}\left[f\left(q_{2}, r\right)-f\left(q_{1}, r\right)\right] .
\end{gathered}
$$

Here we have introduced the values $q_{1}=\xi^{-1}\left(\sqrt{\sqrt{1+w^{2}}-1}+\right.$ $\left.i \sqrt{\sqrt{1+w^{2}}+1}\right), q_{2}=\xi^{-1} \sqrt{2 i w}, w=\omega \tau / 2$, and

$$
f(q, r)=\frac{J_{1}(q r)}{q R J_{0}(q R)-J_{1}(q R)},
$$

where $J_{0}$ and $J_{1}$ are the Bessel functions.

The obtained complex amplitudes enable us to calculate the spatial profile of the dc superconducting current,

$$
\left\langle j_{s \theta}(r)\right\rangle=\frac{4 \pi \alpha T_{c} \xi_{0}^{2} c}{\Phi_{0}} \Delta_{0} \operatorname{Re}\left\{\tilde{\Delta}_{1}\left(\frac{i \tilde{\chi}^{*}}{r}-\frac{2 \pi}{\Phi_{0}} \tilde{A}_{\theta}^{*}\right)\right\} .
$$

After substitution and simplification, we obtain

$$
\begin{aligned}
\left\langle j_{s \theta}(r)\right\rangle= & \frac{\gamma}{\alpha} \frac{32 e^{3} \Delta_{0}^{2} E_{0}^{2} \tau^{2}}{\pi^{2} \alpha m^{2} \omega R}\left(\frac{R}{\xi}\right)^{2} \\
& \times \operatorname{Re}\left\{\left[f\left(q_{1}, r\right)-f\left(q_{2}, r\right)\right]\left[1-(R / r) f\left(q_{2}^{*}, r\right)\right]\right\},
\end{aligned}
$$

where $m=\hbar^{2} /\left(4 \alpha T_{c} \xi_{0}^{2}\right)$ is the mass characterizing the Cooper pair.

The typical profiles of the dc current in the disk are shown in Fig. 2. Remarkably, for relatively large disks the current changes its direction at certain distances from the center. Qualitatively, this phenomenon is associated with the coupled oscillations of the amplitude and phase of the superconducting order parameter [see Eqs. (6)-(8)]. Note that in the limit $\gamma \gg \alpha$ these oscillations take the form of the 

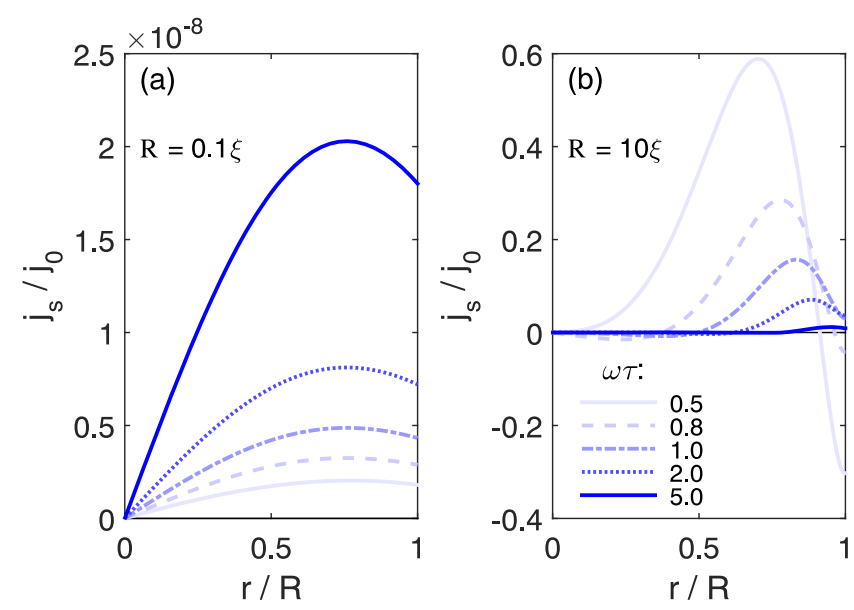

FIG. 2. Dependencies of the azimuthal superconducting current as a function of the distance $r$ from the disk center for (a) $R=0.1 \xi$ and (b) $R=10 \xi$. Different curves correspond to different radiation frequencies. The values of the parameter $\omega \tau$ relevant for both panels are shown in (b). We denote $j_{0}=32 \gamma e^{3} \Delta_{0}^{2} E_{0}^{2} \tau^{3} /\left(\pi^{2} \alpha^{2} m^{2} \xi\right)$.

soundlike waves similar to the Bogoliubov modes in the Bose-Einstein condensate (see, e.g., [23]). The oscillations of the order parameter amplitude are responsible for the appropriate contribution to the supercurrent and it is the sign change of the correction $\Delta_{1}$ that determines the sign change to the supercurrent (12). At the same time, the total magnetic moment of the disk $M=(L / c) \int_{0}^{R}\left\langle j_{s \theta}\right\rangle \pi r^{2} d r$ (where $L$ is the disk thickness) is fixed by the light polarization (see the Supplemental Material [22] for the analytical expression). The typical dependencies of the total magnetic moment $M$ on the radiation frequency $\omega$ for different ratios $(R / \xi)$ are shown in Fig. 3.

For small disks with $R \ll \xi$, the magnetic moment $M$ linearly grows as a function of $\omega$ in the low-frequency limit and reveals a crossover to $M \propto \omega^{-3}$ behavior at $\omega \tau \sim(\xi / R)^{2}$,

$M=\frac{\gamma}{\alpha} \frac{64 e^{3} \Delta_{0}^{2} E_{0}^{2} L R^{2}}{\pi \alpha m^{2} c} \begin{cases}\beta \omega \tau^{4}\left(\frac{R}{\xi}\right)^{8}, & \text { for } \omega \tau \ll\left(\frac{\xi}{R}\right)^{2}, \\ \frac{1}{\omega^{3}}, & \text { for } \omega \tau \gg\left(\frac{\xi}{R}\right)^{2},\end{cases}$

where $\beta=73 / 11520 \approx 6 \times 10^{-3}$.

In the opposite limit, when $R \gg \xi$, the dependence $M(\omega)$ is characterized by three regimes

$$
M=\frac{\gamma}{\alpha} \frac{64 e^{3} \Delta_{0}^{2} E_{0}^{2} L R^{2}}{\pi \alpha m^{2} c} \begin{cases}\eta \omega \tau^{4}\left(\frac{R}{\xi}\right)^{4}, & \text { for } \omega \tau \ll\left(\frac{\xi}{R}\right)^{2}, \\ \frac{\tau^{2}}{4 \omega}, & \text { for }\left(\frac{\xi}{R}\right)^{2} \ll \omega \tau \ll 1, \\ \frac{1}{\omega^{3}}, & \text { for } \omega \tau \gg 1,\end{cases}
$$

where $\eta=7 / 384 \approx 0.018$.
Note that Eq. (4) is valid for the radiation frequency $\omega$ smaller than the characteristic frequencies of the inelastic electron-phonon relaxation processes $\tau_{\mathrm{ph}}^{-1}$ [20]. At the same time, the GL relaxation time $\tau$ becomes infinitely large when $T$ approaches $T_{c}$. Thus, even in the limit $\omega \ll \tau_{\mathrm{ph}}^{-1}$ the parameter $\omega \tau$ in (14) and (15) can take the values both greater and smaller than unity.

The above contribution to the magnetic moment $M$ of the disk should be, of course, summed up with the term arising from the Hertel contribution associated with the nonsuperconducting electrons [8].

It is important to note that the electric field $\mathbf{E}$ acting on the electrons inside the superconducting disk does not coincide with the electric field $\mathbf{E}^{\text {ext }}$ of the incident electromagnetic wave due to the depolarization effects. The simplified relation between these quantities can be established by approximating the disk with the conducting ellipsoid of the semiaxes $R, R$, and $L / 2$. In this case, one gets [18]

$$
\mathbf{E}=\frac{\mathbf{E}^{\mathrm{ext}}}{1+(\pi / 8)(L / R)[\varepsilon(\omega)-1]},
$$

where $\varepsilon(\omega)$ is the frequency-dependent permittivity of the superconducting metal. In the collisionless limit $\varepsilon(\omega)=1-\omega_{p}^{2} / \omega^{2}$, where $\omega_{p}=\left(4 \pi e^{2} N_{0} / m\right)^{1 / 2}$ is the electronic plasma frequency of the metal. As a result, in the low-frequency limit, when $\omega \ll \omega_{p} \sqrt{L / R}$ in Eq. (3), one finds $|\mathbf{E}|^{2} \sim\left(\omega / \omega_{p}\right)^{4}(R / L)^{2}\left|\mathbf{E}^{\text {ext }}\right|^{2}$. This additional factor coming from the depolarization effects provides a natural cutoff for expression (3) in the limit $\omega \rightarrow 0$. Because of this renormalization, the magnetization arising in normal metals due to the IFE (see [8]) should also vanish at zero frequency.

To estimate the possible values of the optically induced magnetic moment for simplicity, let us consider the case $R \gtrsim \xi$ and $\omega \tau \gg 1$. Then, accounting for $\gamma / \alpha \sim T_{c} / E_{F}$ [21] and $\alpha \sim T_{c} / E_{F}$ from expression (15), we obtain

$$
M \sim 10 \mu_{B} \frac{\Delta_{0}^{2}}{N} \frac{P}{\hbar \omega^{2}}
$$

Here $\mu_{B}=e \hbar /(2 m c)$ is the Bohr magneton, $P=$ $\left(\omega_{p}^{2} / \omega\right)\left(|\mathbf{E}|^{2} V / 4 \pi\right)$ is the total power absorbed inside the superconducting disk, $V=\pi R^{2} L$ is the disk volume, and $\omega_{p}=\left(4 \pi e^{2} N / m\right)^{1 / 2}$ is the electron plasma frequency. The relation between $P$ and the electromagnetic wave intensity $\mathcal{I}=c\left|\mathbf{E}^{\text {ext }}\right|^{2} / 8 \pi$ is determined by the sample geometry and the radiation frequency. For $\omega^{2} \ll \omega_{p}^{2}(L / R)$ the depolarization effects significantly influences the electric field inside the disk so that $|\mathbf{E}| \sim\left|\mathbf{E}^{\mathrm{ext}}\right|\left(\omega^{2} / \omega_{p}^{2}\right)(R / L)$. As a result, at temperatures $T \ll T_{c}$ we may put $\Delta_{0}^{2} \approx N$ and finally obtain 

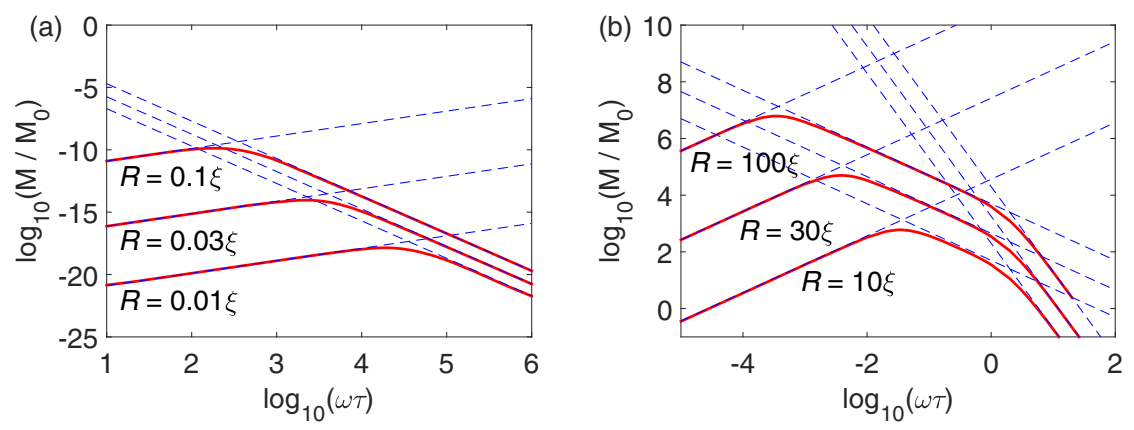

FIG. 3. Dependence of the magnetic moment on the radiation frequency for the disks with (a) $R \ll \xi$ and (b) $R \gg \xi$. Here $M_{0}=8 \pi^{4} \gamma \hbar \tau \xi^{6} c^{2} L \Delta_{0}^{2} E_{0}^{2} / \Phi_{0}^{3}$.

$$
M \sim 10 \mu_{B} \frac{\omega^{2}}{\omega_{p}^{2}} \frac{R^{2}}{L^{2}} \frac{\mathcal{I} V}{\hbar \omega c} .
$$

Taking the disk of the radius $R \sim 10 \mu \mathrm{m}$ and the thickness $L \sim 10 \mathrm{~nm}$ radiated by the infra-red wave with $\omega \sim$ $10^{13} \mathrm{sec}^{-1}$ and intensity $\mathcal{I} \sim 10 \mu \mathrm{W} / \mu \mathrm{m}^{2}$, and taking $\omega_{p} \sim$ $10^{15} \mathrm{sec}^{-1}$ (keeping in mind high- $T_{c}$ cuprates) we can get $M \sim 10^{3} \mu_{B}$.

Of course, choosing the appropriate laser beam intensity one needs to make sure that the heating does not destroy the superconducting state. To arrange the effective heat removal one can, e.g., place the superconducting film on top of the sapphire substrate. Thanks to the very high thermal conductivity of sapphire $\kappa \sim 10^{3} \mathrm{~W} /(\mathrm{m} \mathrm{K})$ at $T \sim 10 \mathrm{~K}$ [24], the substrate of the thickness $d_{\text {sub }} \sim$ $1 \mu \mathrm{m}$ with the temperature elevation of $\Delta T \sim 1 \mathrm{~K}$ between its edges can support the heat transfer of the surface power density up to $q \sim \kappa \Delta T / d_{\text {sub }} \sim 10^{3} \mu \mathrm{W} / \mu \mathrm{m}^{2}$. Thus, even in the case of the full absorption of the laser radiation of the intensity $\mathcal{I} \sim 10 \mu \mathrm{W} / \mu \mathrm{m}^{2}$, the disk on top of the substrate remains superconducting. The experimental achievability of such regime was demonstrated, e.g., in Ref. [25], where under the influence of the laser beam with the absorbed power $17 \mu \mathrm{W}$ the temperature of $\mathrm{Nb}$ film of thickness $90 \mathrm{~nm}$ placed on the $500 \mu \mathrm{m} \mathrm{Si}$ substrate did not exceed $T_{c}$.

Clearly, the predicted phenomena should become dramatically enhanced in superconducting media where $T_{c} / E_{F} \sim 1$ (e.g., Bose-Einstein condensates in the local pairs condensation regime [26] or the superconductors with extremely small electron density like $\mathrm{SrTiO}_{3}$ [27]). In such systems, the superconducting currents induced by the circularly polarized light may become large enough to generate vortices that can survive due to the presence of the pinning effects even after the laser pulse is switched off.

Finally, we note that the measurements of the IFE contribution caused by the gap modulation provide an interesting possibility to establish the connection between three different phenomena: the IFE, direct Faraday effect, and Hall effect in the vortex state. Indeed, the imaginary part of the relaxation constant $\gamma$ is known to affect the vortex contribution to the off-diagonal component $\sigma_{x y}$ of the complex conductivity tensor that controls the rotation of the light polarization plane due to the direct Faraday effect $[28,29]$. The renormalization of $\sigma_{x y}$ due to the nonzero $\gamma$ can be also responsible for the anomalous sign change of the Hall coefficient near the superconducting critical temperature for a number of superconducting compounds including high $-T_{c}$ cuprates. The sign of the $\gamma$ parameter thus determines both the sign of the contributions to the induced magnetic moment in the IFE and to the Hall conductivity governed by the gap dynamics.

This work was supported by the Russian Science Foundation (Grant No. 18-72-10027) in part related to the calculation of magnetic moment arising due to the IFE and the Russian Foundation for Basic Research (Grant No. 18-02-00390). S. M. acknowledges the financial support of the Foundation for the Advancement of Theoretical Physics and Mathematics BASIS (Grant No. 18-1-3-58-1) and Russian Presidential Scholarship SP-3938.2018.5. A.S.M. acknowledges support from the Bordeaux University IDEX program. The work of A. B. and B. L. was supported by the French ANR OPTOFLUXONICS, EU COST CA16218 Nanocohybri.

[1] L. P. Pitaevskii, JETP 12, 1008 (1961).

[2] J. P. van der Ziel, P. S. Pershan, and L. D. Malmstrom, Phys. Rev. Lett. 15, 190 (1965).

[3] A. Kirilyuk, A. V. Kimel, and T. Rasing, Rev. Mod. Phys. 82, 2731 (2010).

[4] A. Kirilyuk, A. V. Kimel, and T. Rasing, Rep. Prog. Phys. 76, 026501 (2013).

[5] V. Kimel, A. Kirilyuk, P. A. Usachev, R. V. Pisarev, A. M. Balbashov, and Th. Rasing, Nature (London) 435, 655 (2005).

[6] C. D. Stanciu, F. Hansteen, A. V. Kimel, A. Tsukamoto, A. Itoh, A. Kirilyuk, and Th. Rasing, Phys. Rev. Lett. 98, 207401 (2007).

[7] O. H.-C. Cheng, D. H. Son, and M. Sheldon, Nat. Photonics 14, 365 (2020).

[8] R. Hertel, J. Magn. Magn. Mater. 303, L1 (2006).

[9] R. Hertel and M. Fähnle, Phys. Rev. B 91, 020411(R) (2015). 
[10] M. Battiato, G. Barbalinardo, and P. M. Oppeneer, Phys. Rev. B 89, 014413 (2014).

[11] I. D. Tokman, Phys. Lett. A 252, 83 (1999).

[12] G. F. Quinteiro and P. I. Tamborenea, Europhys. Lett. 85, 47001 (2009).

[13] K. L. Koshelev, V. Yu. Kachorovskii, and M. Titov, Phys. Rev. B 92, 235426 (2015).

[14] O. V. Kibis, Phys. Rev. Lett. 107, 106802 (2011).

[15] C. Ryu, M. F. Andersen, P. Cladé, Vasant Natarajan, K. Helmerson, and W. D. Phillips, Phys. Rev. Lett. 99, 260401 (2007).

[16] S. Beattie, S. Moulder, R. J. Fletcher, and Z. Hadzibabic, Phys. Rev. Lett. 110, 025301 (2013).

[17] J. Linder and J. W. A. Robinson, Nat. Phys. 11, 307 (2015).

[18] L. D. Landau, L. P. Pitaevskii, and E. M. Lifshitz, Electrodynamics of Continuous Media (Elsevier, New York, 1984).

[19] L. Bulaevskii, A. Buzdin, M. Kulic, and S. Panjukov, Adv. Phys. 34, 175 (1985).

[20] N. Kopnin, Theory of Nonequilibrium Superconductivity (Oxford Science, London, 2001).

[21] A. I. Larkin and A. A. Varlamov, in Physics of Conventional and Nonconventional Superconductors, edited by
K.-H. Bennemann and J. B. Ketterson (Springer-Verlag, Berlin, 2002).

[22] See Supplemental Material at http://link.aps.org/ supplemental/10.1103/PhysRevLett.126.137002 for the detailed calculation of the light-induced dc current and magnetic moment.

[23] C. J. Pethick and H. Smith, Bose-Einstein Condensation in Dilute Gases (Cambridge University Press, Cambridge, England, 2002).

[24] R. Berman, E. L. Foster, and J. M. Ziman, Proc. R. Soc. A 231, 130 (1955).

[25] I. S. Veshchunov, W. Magrini, S. V. Mironov, A. G. Godin, J.-B. Trebbia, A. I. Buzdin, Ph. Tamarat, and B. Lounis, Nat. Commun. 7, 12801 (2016).

[26] W. Ketterle, Y. Shin, A. Schirotzek, and C. H. Schunk, in BCS: 50 Years, edited by L. N. Cooper and D. Feldman (World Scientific, Singapore, 2010).

[27] X. Lin, Z. Zhu, B. Fauqué, and K. Behnia, Phys. Rev. X 3, 021002 (2013).

[28] A. T. Dorsey, Phys. Rev. B 46, 8376 (1992).

[29] N. B. Kopnin, B. I. Ivlev, and V. A. Kalatsky, Pis'ma Zh. Eksp. Teor. Fiz. 55, 717 (1992) [JETP Lett. 55, 750 (1992)]. 